\title{
Atomic data for integrated tokamak modelling - Fermi-shuttle type ionization as a possible source of high energy electrons
}

\author{
K. Tőkési ${ }^{1, \mathrm{a}}$, D. Tskhakaya ${ }^{2, \mathrm{~b}}$ and D. Coster ${ }^{3}$ \\ ${ }^{1}$ Institute for Nuclear Research, Hungarian Academy of Sciences (ATOMKI), Debrecen, Hungary \\ 2 Association EURATOM-ÖAW, Inst. of Applied Physics, TU Wien, 1040 Vienna, Austria \\ ${ }^{3}$ Max-Planck-Institut für Plasmaphysik, 85748 Garching, Germany
}

\begin{abstract}
The ionization of Ar by $15 \mathrm{keV} \mathrm{N}^{+}$ion is studied theoretically. The energy distributions of the ejected electrons as a function of the scattering angle were calculated using the classical trajectory Monte Carlo method. We identify the signature of the Fermishuttle type ionization in the double differential cross sections which should be a possible source of the high energy electrons in the plasma. Our classical calculation also describes the previously measured data with high accuracy.
\end{abstract}

\section{Introduction}

The Integrated Tokamak Modeling Task Force (ITM-TF) was set up in 2004. The main target is to coordinate the European fusion modeling effort and providing a complete European modeling structure for International Thermonuclear Experimental Reactor (ITER) and other future magnetic confinement devices (e.g. DEMO) with the highest degree of flexibility. One the ITM-TF projects is the collection, verification and implementation of the atomic, molecular, nuclear and surface related data relevant for fusion.

Within the framework of ITM-TF we performed classical simulations for a various collision systems. In this work we present double ionization cross sections in close connection to the ITM-TF. The main aim of the present work is to find the possible source of generation of the high energy electrons in the plasma. At sufficiently low collision energies, it is known that the so-called Fermi-shuttle type ionization mechanism can produce high energy electrons [1,2]. Originally, Fermi proposed the mechanism as a possible source of energetic cosmic rays [3] where weak but giant magnetic fields, moving in outer space, can accelerate charged particles with extremely high energies in long sequences of reflection. In this case the number of collisions is approximately $10^{8}$ and the particles gain approximated 10 $\mathrm{eV}$ energy at each collisions. Similarly, a light electron can be scattered forward and backward by heavy target atoms and an incoming projectile ion before being ejected. With heavy atomic centers. $\left(M / m_{e} \gg 1\right.$, where $\mathrm{M}$ is the mass of the target or projectile and $m_{e}$ is the mass of the electron), the kinematics of collision is very simple. Taken into account the energy and momentum conservation, the

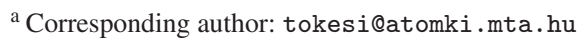

${ }^{\mathrm{b}}$ Permanent address: Andronikashvili Institute of Physics, 0177 Tbilisi, Georgia
}

This is an Open Access article distributed under the terms of the Creative Commons Attribution License 4.0, which permits unrestricted use, distribution, and reproduction in any medium, provided the original work is properly cited. 
velocity of the liberated electron is increased by approximately $2 \mathrm{~V}$, in every $180^{\circ}$ elastic scattering with the incoming projectile, while only the direction of the electronic motion is changed by scatterings on the target field. Due to the repeated collisions, the active electron can be accelerated to relatively high energies. After 1, 2, 3, 4, or 5 collisions the electron velocity increased approximated by $2 \mathrm{~V}, 4 \mathrm{~V}, 6 \mathrm{~V}$, $8 \mathrm{~V}$ and $10 \mathrm{~V}$, where $\mathrm{V}$ is the velocity of the projectile. Therefore the corresponding energy gain is $4 \mathrm{E}_{\mathrm{V}}$, $16 \mathrm{E}_{\mathrm{V}}, 36 \mathrm{E}_{\mathrm{V}}, 64 \mathrm{E}_{\mathrm{V}}, 100 \mathrm{E}_{\mathrm{V}}$, where $\mathrm{E}_{\mathrm{V}}=0.5 m_{e} \mathrm{~V}^{2}$. Hence, although naturally in atomic level the number scattering is much less compared with the case of giant magnetic field, but even after a few back and forth collisions the active electron can gain enormous energy.

In this work as an example we present electron emission cross sections in collision between dressed $\mathrm{N}^{+}$ions with Ar target at $15 \mathrm{keV}$ impact energy. The theory delivers separate spectra for electrons emitted from the target and the projectile. By summing these two components in the rest frame of the target we may also make a comparison with available experimental data also.

\section{Theory}

Interpretation of the Fermi-shuttle-type measurements is a challenging task for theories. The main difficulty is caused by the many-body feature of the collision, involving the projectile, projectile electron(s), target nucleus, and target electron(s). The classical trajectory Monte Carlo (CTMC) method has been quite successful in dealing with the ionization process in ion-atom collisions. One of the advantages of the CTMC method is that many-body interactions are exactly taken into account during the collisions on a classical level.

The CTMC method is a nonperturbativ method, where classical equations of motions are solved numerically [4-9]. In the present work the CTMC simulations were made in the three-body approximation, i.e. the many-electron target atom was replaced by a one-electron atom and the projectile ion was taken into account as one particle $[8,9]$. Therefore in our CTMC model the three particles are the projectile $(\mathrm{P})$, one atomic active target electron (e), and the remaining target ion $(\mathrm{T})$, including the target nucleus and the remainder target electrons.

The three particles are characterized by their masses and charges. We note that this model is the classical analogue of the quantum-mechanical effective single-electron treatment of the collisions in which the electrons are treated equivalently. Both the target atom-electron and the projectile ion-electron potentials are represented by central model potentials developed by Green [10] whose parameters are derived from Hartree-Fock calculations. The potential can be written as:

$$
V(r)=q \frac{Z-(N-1)\left(1-\Omega^{-1}(r)\right)}{r}=q \frac{Z(r)}{r},
$$

where $Z$ is the nuclear charge, $N$ is the total number of electrons in the atom or ion, $r$ is the distance between the nucleus and the test charge $q$, and

$$
\Omega(r)=\frac{\eta}{\xi}\left(e^{r \xi}-1\right)+1 .
$$

The potential parameters $\xi$ and $\eta$ can be obtained in such a way that they minimize the energy for a given atom or ion. We note that this type of potential has further advantages, because it has a correct asymptotic form for both small (Eq. (3)) and large (Eq. (4)) values of $r$.

$$
\begin{gathered}
\lim _{r \rightarrow 0} Z(r)=Z \\
\lim _{r \rightarrow \infty} Z(r)=Z-(N-1) .
\end{gathered}
$$

Although this potential was originally developed for describing the electronic ground states, we will use this form to mimic of the distance dependent charge of the dressed projectile also. 
The initial conditions of an individual collision are chosen at sufficiently large inter-nuclear separations from the collision center, where the interactions among the particles are negligible. These conditions, which are constrained to the initial binding energy of the active target electron, are selected in a similar fashion as described by Reinhold and Falcon [11] for non-Coulomb systems. A microcanonical ensemble characterizes the initial state of the target. The classical equations of motion were integrated with respect to time as an independent variable by the standard Runge-Kutta method. To distinguish between the various final states, the exit channels are identified at large distances from the collision center. The doubly differential cross sections were computed using the following formula:

$$
\frac{d^{2} \sigma}{d E d \Omega}=\frac{2 \pi b_{\max }}{T_{N} \Delta E \Delta \Omega} \sum_{j} b_{j}^{(i)} .
$$

The statistical uncertainty of the cross section is given by

$$
\Delta \sigma=\sigma\left(\frac{T_{N}-T_{N}^{(i)}}{T_{N} T_{N}^{(i)}}\right)^{1 / 2} .
$$

In Eqs. (1) and (2) $T_{N}$ is the total number of trajectories calculated for impact parameters less than $b_{\max }, T_{N}^{(i)}$ is the number of trajectories that satisfy the criteria for ionization, and $b_{j}^{(i)}$ is the actual impact parameter for the trajectory corresponding to the ionization process under consideration in the energy interval $\Delta E$ and the emission angle interval $\Delta \Omega$ of the electron.

\section{Results}

We performed CTMC calculations for the collision system of $\mathrm{N}^{+}+\mathrm{Ar}$ at $15 \mathrm{keV}$ impact energy to determine the ionization cross sections as a function of emission angle and energy of the ejected electrons in order to understand how the electrons gain energy during the possible multiple scattering between the projectile and the target nucleus. The system is a challenge to describe theoretically owing to screening of the projectile nuclear charge by bound electrons, interactions of projectile electrons with target electrons, and the large number of possible exit channels. For the collision system in question, a significant contribution from Fermi-shuttle ionization [1-3] has to be expected in the spectra at energies higher than $E=0.5 m_{e}(n V)^{2}$, where $\mathrm{m}_{\mathrm{e}}$ is the mass of the electron, $V$ the projectile velocity and $n$ an integer greater than 1 .

Figure 1 shows the doubly differential cross sections (DDCS) for ejection of electrons in $15 \mathrm{keV}$ $\mathrm{N}^{+}-$Ar collisions at $30^{\circ}, 90^{\circ}$, and $135^{\circ}$. Since no structure appeared in the energy spectra, like at higher energies was obtained $[1,2]$, we considered this fact as an indication for overlapping multiple scattering $(n V)$ contributions. At the same time, we obtained significant electron emission yields at the binary encounter peak $(2 \mathrm{~V})$ and even higher energies in the energy range belonging to the $6 \mathrm{~V}-14 \mathrm{~V}$ electron velocity range. We found electron yields even for $n=14$ of CTMC spectra. This is a clear indication for the existence of longer (6-14) multiple scattering sequences which can be directly attributed to the contribution of Fermi-shuttle type multiple scattering producing fast.

Figure 2 shows the measured spectrum of the electrons emitted at $135^{\circ}$ observation angle with the recent CTMC results. Since the measured electron emission cross sections are not absolute, the experimental data are normalized to the CTMC value at $40 \mathrm{eV}$.

Our CTMC calculations reproduced quite well the main features of the experimental spectra. The disagreement at low and high energies is caused that the recent theory is not taken into account the electron emission from Auger processes. The angular dependence of the collision is correctly accounted for (not shown). We note that almost all CTMC electron emission "events" above $10 \mathrm{eV}$ fall into the multiple scattering category indicating that accelerating multiple scattering can dominate electron emission at low ion impact energies. 


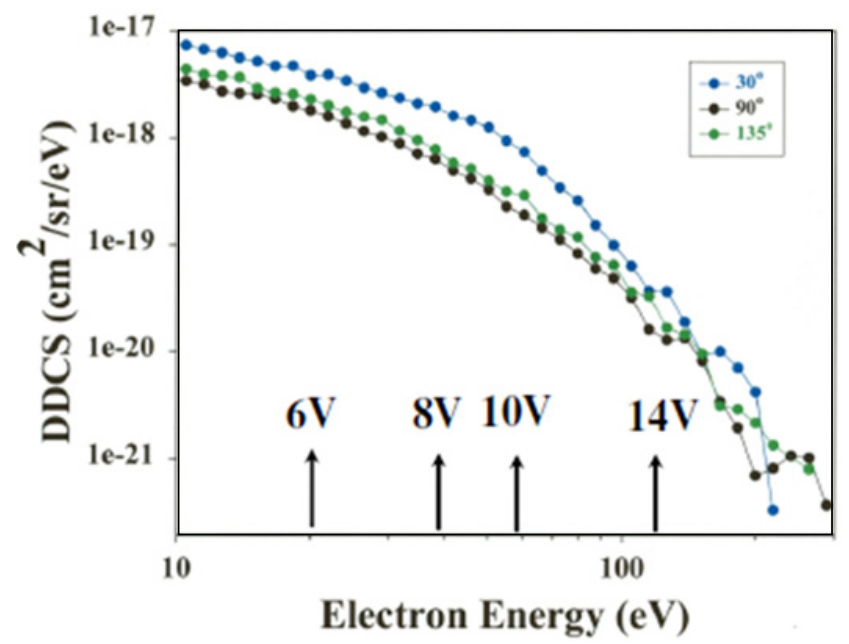

Figure 1. Energy distribution of electrons ejected in collision between $15 \mathrm{keV} \mathrm{N}^{+}$ions and Ar atoms at $30^{\circ}, 90^{\circ}$, and $135^{\circ}$. CTMC data contain contribution from both the target and projectile ionization. Multiples of the projectile velocities are shown.

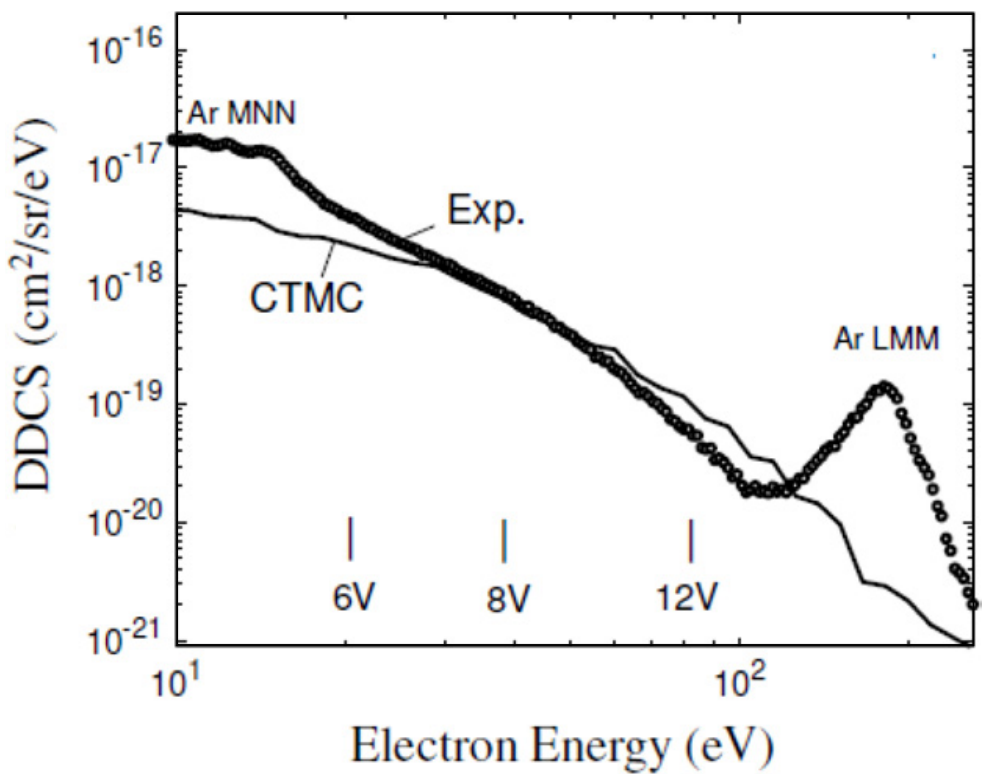

Figure 2. Energy distribution of electrons ejected in collision between $15 \mathrm{keV} \mathrm{N}^{+}$ions and Ar atoms at $135^{\circ}$. Circle: experiment [12], Solid line: CTMC data, contribution from both the target and projectile ionization. Multiples of the projectile velocities are shown.

\section{Conclusion}

We have shown that the classical treatment of atomic collisions reproduce the emitted electron energy spectrum of $15 \mathrm{keV} \mathrm{N}^{+}$ions interacting with argon. The signature of the Fermi shuttle type ionization can be identified in the electron spectra, thus indicating that this multiple scattering mechanism is important for describing the energetic electrons produced in low energy ion-matter interactions. These 


\section{$3^{\text {rd }}$ European Energy Conference}

electrons can significantly contribute for further excitation sin the plasma. The corresponding data will be implemented into the ITM data structure.

This work, supported by the European Communities under the contract of Association between EURATOMHAS, was carried out within the framework of the Task Force on Integrated Tokamak Modeling of the European Fusion Development Agreement. The views and opinions expressed herein do not necessarily reflect those of the European Commission. The work was also supported by the Hungarian Scientific Research Fund OTKA No. NN103279.

\section{References}

[1] B. Sulik, Cs. Koncz, K. Tőkési, A. Orbán, D. Berényi, Phys. Rev. Lett. 88 (2003) 073201

[2] B. Sulik, Cs. Konc, K. Tôkési, Á. Kövér, S. Ricz, Gy. Víkor, J.-Y. Chesnel, N. Stolterfoht, D. Berényi, Nucl. Instr. and Meth. B 154 (1999) 281

[3] E. Fermi, 1949 Phys. Rev. 75, 1169

[4] R. Abrines and I.C. Percival 1966 Proc Phys Soc (London) 88, 861

[5] R.E. Olson and A. Salop, Phys. Rev. A16 (1977) 531

[6] K. Tőkési, G. Hock, Nucl. Instrum Meth. in Phys. Res. B86 (1994) 201

[7] K. Tôkési, G. Hock, J. Physics B 29 (1996) 119

[8] K. Tőkési and Á.Kövér 1999 Nucl. Instrum Meth. in Phys. Res. B154, 259

[9] K. Tőkési, Á. Kövér, J. Phys. B 33 (2000) 3067

[10] A.E.S Green 1973 Adv Quantum Chem. 7, 221

[11] C.O. Reinhold and C.A. Falcon 1986 Phys. Rev. A33, 3859

[12] B. Sulik, Cs. Konc, K. Tőkési, Á. Kövér, S. Ricz, Gy. Víkor, J.-Y. Chesnel, N. Stolterfoht and D. Berényi, Nucl. Inst. Meth. B 154 (1999) 281 